
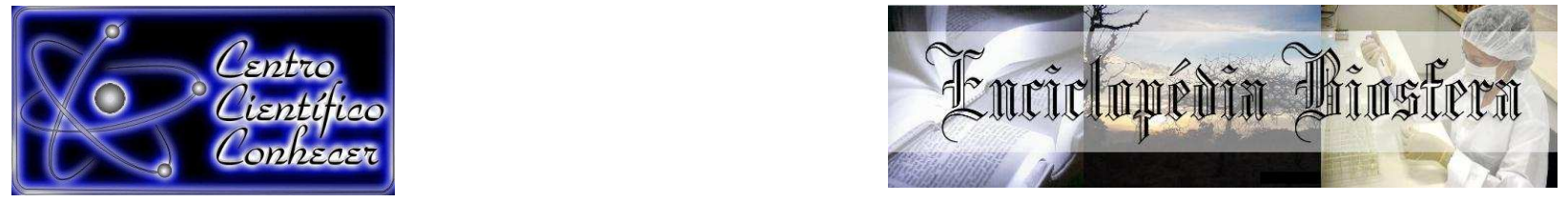

\title{
EFICÁCIA DA DELTAMETRINA NO CONTROLE DE LARVAS DE Amblyomma cajennense (FABRICIUS, 1787) (ACARI: IXODIDAE).
}

Luciano Melo de Souza ${ }^{1}$, Rafael Molena Ambrozio², Marco Antonio de Andrade Belo $^{1,4}$, Ives Charlie da Silva ${ }^{3}$, Denis Chiquito ${ }^{2}$

${ }^{1}$ Professor Doutor - Titular de Parasitologia e Doenças Parasitárias da Universidade Camilo Castelo Branco (lucianomelos@gmail.com), Descalvado, São Paulo, Brasil.

${ }^{2}$ Acadêmico do curso de Medicina Veterinária da Universidade Camilo Castelo

Branco, Descalvado, São Paulo, Brasil.

${ }^{3}$ Doutorando da Universidade Estadual Paulista Júlio de Mesquita Filho, Jaboticabal, São Paulo, Brasil.

${ }^{4}$ Professor Doutor do Departamento de Medicina Veterinária Preventiva da Universidade Estadual Paulista Júlio de Mesquita Filho, Jaboticabal, São Paulo, Brasil.

\section{Recebido em: 08/09/2015 - Aprovado em: 14/11/2015 - Publicado em: 01/12/2015 DOI: http://dx.doi.org/10.18677/Enciclopedia_Biosfera_2015_148}

\begin{abstract}
RESUMO
Testou-se in vitro a eficácia de carrapaticidas sobre larvas do Amblyomma cajennense com os seguintes tratamentos: Controle - água destilada; Amitraz 12,5\%; Associação - Cipermetrina 15\% + Clorpiriphos 25\% + Citronela 1\%; Amitraz 12,5\%; Cipermetrina 15\%; e Deltametrina 25\%; utilizando-se as técnicas da pipeta de Pasteur Impregnada (TPPI) e do Pacote de Larvas (TPL). Empregaram-se dosagens prescritas pelos fabricantes para os fármacos em dois experimentos. Experimento I: Verificou-se a eficácia antiparasitária através da perda de motilidade das larvas pela TTPI em observações nos tempos 5', 60', 120', 6, 12 e 24 horas após tratamento (HPT). Larvas tratadas com amitraz $12,5 \%$ apresentaram significativa perda de motilidade na fase aguda, alcançando eficácia superior a 90\% nos 5' e 60' após o tratamento, porém apenas $56,5 \%$ das larvas tratadas com amitraz $12,5 \%$ apresentavam perda de motilidade 24 HPT, confirmando o efeito tóxico transitório deste composto. Larvas expostas ao tratamento com deltametrina $25 \%$ apresentaram eficácia tardia significativa, resultando em $88,5 \%$ de larvas com perda de motilidade $24 \mathrm{HPT}$. Experimento II: Com o TPL observou-se os respectivos percentuais de mortalidade larvar: 90\% (Cipermetrina 15\% + Clorpirifós 25\% + Citrolena 1\%), 73,3\% (Deltametrina 25\%), 56,6\% (Cipermetrina 15\%) e 43,3\% (Amitraz 12,5\%) 24 HPT. A eficácia do tratamento com deltametrina $25 \%$ sobre a motilidade de larvas deste ixodídeo confirma sua importância para o controle ambiental do instar. O tratamento com amitraz 12,5\% apresentou efeito tóxico transitório sobre a motilidade das larvas, ocorrendo recuperação da capacidade motora não sendo recomendado para o uso no controle ambiental deste ácaro.
\end{abstract}

PALAVRAS-CHAVE: carrapato, equinos, ixodídeos, resistência, zoonoses. 


\title{
DELTAMETHRIN EFFECTIVENESS IN THE CONTROL OF Amblyomma cajennense (FABRICIUS, 1787) (ACARI: IXODIDAE) LARVAE.
}

\begin{abstract}
This study aimed to test in vitro the efficacy of different acaricides on larvae of Amblyomma cajennense with the following treatments: Control treated with distilled water; Amitraz 12.5\%; Association of Clorpiriphos Cypermethrin 15\% $+25 \%+1 \%$ Citronella; Amitraz 12.5\%; Cypermethrin 15\%; and Deltamethrin 25\%; using the Pipette Technique of Pasteur Impregnated and Larvae Pack Test (LPT) recommended by the United Nations Food and Agriculture Organization (FAO). The dosages prescribed were tested by manufacturers for different drugs in two experiments. Experiment I - To ascertain antiparasitic efficacy by paralysis and loss of larval motility in the pipette technique were performed 5 ', 60', 120 ', 6, 12 and 24 hours after treatment (HPT). Larvae treated with amitraz $12.5 \%$ had a significant loss of motility in the acute phase, achieving greater efficacy than $90 \%$ during periods of 5 and 60 minutes after treatment, but only $56.5 \%$ of the larvae treated with amitraz $12.5 \%$ presented motilityt $24 \mathrm{HPT}$, confirming the transitional toxic effects of this compound. Larvae exposed to treatment with deltamethrin $25 \%$ were significantly effectiveness resulting in $88.5 \%$ of larvae with loss of motility 24 HPT. Experiment II The larvae test package was observed for the corresponding percentage of larval mortality: $90 \%(15 \%$ Cypermethrin + Chlorpyrifos Citrolena 25\% + 1\%) $73.3 \%$ (Deltamethrin 25\%), 56.6\% (Cypermethrin 15\%) and 43.3\% (12.5\% Amitraz) $24 \mathrm{PTH}$. The efficacy of deltamethrin $25 \%$ on the larval motility confirms the importance of this compound for the environmental control larvae. However, treatment with amitraz $12.5 \%$ had a transient toxic effect on the motility of the larvae occurring recovery of motor ability and thus could not be recommended for use in environmental control of Amblyomma cajennense.
\end{abstract}

KEYWORDS: Ticks, horses, ixodides, tick resistance, zoonosis.

\section{INTRODUÇÃO}

O parasitismo por ácaros ixodídeos permeia a história da humanidade e sua relação com os animais domésticos sempre foi objeto de preocupações, perdas na produção animal e transmissibilidade de agentes etiológicos de importantes doenças animais e zoonóticas. Estes ácaros compreendem aproximadamente 800 espécies conhecidas, todas parasitas de vertebrados terrestres (SOARES et al., 2009).

Dada sua importância na transmissão de doenças, ressaltam-se a seguir as características biológicas do Amblyomma cajennense descritas por BRITES-NETO et al. (2013; 2015). O Amblyomma cajennense completa uma geração por ano, mostrando os três estágios parasitários marcadamente distribuídos ao longo do ano. Esta espécie de carrapato é importante como transmissora da Rickettsia rickettsii, agente etiológico da febre maculosa, a qual é transmitida por via transovariana à sua progênie, o que o torna simultaneamente vetor e reservatório do agente da doença (KRAWCZAK et al., 2014). Esta característica biológica permite ao carrapato permanecer infectado durante toda a sua vida e também por muitas gerações após uma infecção primária, são capazes de permanecer infectadas meses ou anos à espera do hospedeiro, garantindo um foco endêmico prolongado doença (KRAWCZAK et al., 2014).

Casos humanos de febre maculosa têm sido relatados na região Sudeste desde a década de 20, especialmente nos Estados de São Paulo e Minas Gerais. Pelo menos três espécies do gênero Amblyomma ( $A$. cajennense, $A$. aureolatum e $A$. 
cooperi) foram incriminadas de participarem na epidemiologia da febre maculosa no Brasil. No Brasil, é encontrado com abundância em todos os estados das regiões sudeste e centro oeste, porém com distribuição limitada nas demais regiões (PIRES et al., 2013).

O $A$. cajennese é a principal espécie de carrapato que parasita seres humanos no centro-sul brasileiro sendo considerado o principal vetor da febre maculosa brasileira (PIRES et al., 2013; KRAWCZAK et al., 2014). Seus ataques a humanos, muitas vezes em massa, são respondidos com reações imediatas ou, retardadas a antígenos específicos presentes na saliva do carrapato, podendo causar intenso prurido, que persiste por vários dias no local de fixação. Infecções bacterianas secundárias podem ocorrer em função da deposição de bactérias nas feridas, durante ao ato de coçar intensamente (BRITES-NETO et al., 2015).

Inúmeras estratégias são empregadas para controlar carrapatos nos animais. Autores buscam avaliar o controle biológico com fungos entomopatogênicos (BASSO et al., 2005), o uso de fitoterápicos (FARIA et al., 2010; SILVA et al., 2010; TORRENTE et al. 2010), porém, os acaricidas químicos continuam sendo a principal forma de controle dos carrapatos, e forte pressão de seleção imposta, a resistência deles pode ser diagnosticada em grande número de publicações por todo o mundo. No Brasil, pesquisas do controle estratégico do Amblyomma cajennese em equinos utilizando quimioterápicos foram descritas por LABRUNA et al. (2004) e CUNHA et al. (2007), focando principalmente o controle de estágios evolutivos presentes nos animais.

Este uso indiscriminado de formulações indicadas para controle de artrópodes parasitos de bovinos e equinos, para desinsetização de ambientes infestados, e que são comercializadas indevidamente para a descarrapatização, e demais ambientes infestados, inclusive haras, pode corroborar como meio de seleção e indutor de amostras resistentes de parasitos (SOARES et al., 2009; BELO et al., 2012).

De acordo com SOUZA et al. (2009), o controle parasitário é peça fundamental no manejo sanitário e infelizmente tem sido negligenciado pelo técnico de campo existindo um considerável espaço para o envolvimento do Médico Veterinário na implementação de programas de controle parasitário, todavia, ainda observa-se que os profissionais nem sempre fornecem orientações em concordância com as melhores práticas de controle para os criadores. Para estes autores, mesmo com a existência de várias fontes de informação que poderiam auxiliar a tomada de decisões frente ao problema da perda de eficácia das drogas, a maioria dos profissionais não observa a oportunidade de diferenciar o serviço técnico, oferecendo testes diagnósticos antes das prescrições.

A falta de monitoramento da eficácia e uso das drogas no Brasil impossibilita uma análise mais profunda do tema e, provavelmente este seja um dos motivos de existirem inúmeros relatos da redução de atividade dos compostos em vários estados brasileiros (SOARES et al., 2011). Isto revela uma situação tão alarmante quanto a que atualmente ocorre em relação ao carrapato dos bovinos. O problema da resistência parasitária é extremamente sério e compromete diretamente o bemestar dos animais (BELO, et al., 2012). As companhias farmacêuticas têm feito investimentos substanciais nas áreas de pesquisa e desenvolvimento e são partes integrantes do tripé, juntamente com as Universidades, Centros de Pesquisa, técnicos e criadores. Em Descalvado, a ocorrência de infestações graves por Amblyomma cajennense é constante. Em conseqüência deste parasitismo aumentaram-se os casos de enfermidades transmissíveis por este vetor entre os animais domésticos e os seres humanos (BRITES-NETO et al., 2013; 2015). 
Com base na importância epidemiológica do Amblyomma cajennense como vetor e da necessidade de se estabelecer protocolos eficazes de controle de larvas no ambiente e nos animais, este estudo propôs testar in vitro a eficácia de diferentes carrapaticidas sobre larvas deste ixodídeo obtidas de oviposturas de carrapatas colhidas em eqüinos naturalmente infestados da região de Descalvado, utilizando-se a (TPPI) - Técnica da Pipeta de Pasteur Impregnada (SINDIHU et al., 2012; BARCI \& NOGUEIRA, 2006; CASTELLÀ-SPUNY \& SOUZA, 1998) e o (TPL) - Teste do Pacote de Larvas, preconizado pela Organização das Nações Unidas para Agricultura e Alimentação (FAO).

\section{MATERIAL E MÉTODOS}

Material biológico - A colheita de fêmeas ingurgitadas de Amblyomma cajennense para obtenção de larvas foi realizada em haras e criatórios, pertencentes ao município de Descalvado (S21 54 '14 "- W47 ${ }^{\circ} 37^{\prime}$ 12"), localizado no Estado de São Paulo, Brasil.

As amostras foram coletadas de animais que não haviam recebido nenhum tipo de tratamento carrapaticidas tópicos por pelo menos 21 dias e injetáveis pelo menos 45 dias antes da coleta, o que poderia interferir nos resultados. No laboratório, as fêmeas de $A$. cajennense foram separadas de acordo com sua vitalidade e fixadas em placas de Petry para obtenção de ovos. Decorrido o período de postura, as massas produzidas foram recolhidas e armazenadas em seringas de $20 \mathrm{~mL}$ mantidas em estufa climatizada tipo B.O.D. sob temperatura de $27^{\circ} \mathrm{C}$ e $80 \pm$ $10 \%$ de umidade relativa (CHACÓN et al., 2003) para a obtenção dos primeiros instares parasitários (larvas).

Compostos farmacológicos utilizados - As formulações foram diluídas em água destilada utilizando-se provetas volumétricas de $500 \mathrm{~mL}$ cada, respectivamente nas doses indicadas em bulas, pelos fabricantes como mostra na Tabela 1.

TABELA 1. Compostos farmacológicos empregados no estudo de inibição de motilidade de larvas de Amblyomma cajennense.

\begin{tabular}{ccc}
\hline Grupo & Formulação & Diluição \\
\hline I & Água destilada & ---- \\
II & Cipermetrina 15\%+Clorpiriphos 25\%+Citronela 1\% & $0,62 \mathrm{~mL} / 500 \mathrm{~L}$ \\
III & Amitraz 12,5\% & $1 \mathrm{~mL} / 500 \mathrm{~L}$ \\
IV & Cipermetrina 15\% & $1 \mathrm{~mL} / 500 \mathrm{~L}$ \\
V & Deltametrina 25\% & $0,5 \mathrm{~mL} / 500 \mathrm{~L}$
\end{tabular}

GIl: Colosso $\AA^{\circledR}$ - Ouro Fino Saúde Animal, GIII - Triatox® - MSD Saúde Animal, GIV: Cypermil Pulverização® - Ouro Fino Saúde Animal, GV - Butox® - MSD Saúde Animal.

Experimento I - Para a avaliação do efeito toxicológico, indução de desprendimento e queda imediatos (knockdow) dos compostos optou-se pela utilização do método "in vitro" da pipeta de Pasteur, preconizado por BARCI \& NOGUEIRA (2006) e adaptado especificamente para este protocolo experimental. Entre as oviposturas com melhor eclodibilidade (> que $90 \%$ ) as larvas infestantes foram retiradas para os bioensaios frente aos respectivos produtos comercias.

Previamente ao desafio, as pipetas foram impregnadas por imersão em solução contendo os acaricidas devidamente diluídos. Após a secagem das pipetas, 
as larvas selecionadas de acordo com a motilidade foram aspiradas com o auxílio de bomba à vácuo para o interior das pipetas que, após lacradas foram mantidas em estufa vertical climatizada tipo BOD. Em cada tratamento foram realizadas três repetições contendo 20 larvas cada. As observações da motilidade larvar foram realizadas aos 5, 60, 120 minutos, 6, 12 e 24 horas após tratamento, objetivando verificar o efeito imediato knockdown dos fármacos e, posteriormente, até completar 24 horas após o contato com os acaricidas.

Experimento II - Para este ensaio utilizou-se o teste pacote de larvas (TPL), metodologia recomendada pela $\mathrm{FAO}$ e pela portaria № 48 do Ministério da Agricultura, Pecuária e Abastecimento, de 12 de maio de 1997.

Foram delineados tratamentos contendo 100 larvas em três repetições para cada produto. Os papeis filtros foram impregnados conforme a metodologia descrita pela FAO e lacrados em forma de pacotes para que nenhuma larva fugisse do experimento e posteriormente foram incubadas em estufas tipo B.O.D. Para a observação da mortalidade os pacotes foram abertos 24 horas após tratamento e as contagens de larvas vivas e mortas tabuladas para posterior análise.

\section{RESULTADOS E DISCUSSÃO}

\section{Experimento I - Técnica da Pipeta de Pasteur Impregnada}

As observações do efeito farmacológico dos medicamentos testados permitiram compilar dados individualizados nos tempos observacionais propostos para o bioensaio e os resultados estão expressos na Tabela 2.

TABELA 2 - Media percentual da perda de motilidade de larvas submetidas às diferentes formulações carrapaticidas em temos observacionais utilizando-se a técnica da pipeta de Pasteur.

\begin{tabular}{ccccccc}
\hline \multirow{2}{*}{ Tratamento } & $\mathbf{5}$ minutos & $\mathbf{6 0}$ minutos & $\mathbf{1 2 0}$ minutos & $\mathbf{6}$ horas & $\mathbf{1 2}$ horas & 24 horas \\
\cline { 2 - 7 } & Média \% & Média \% & Média \% & Média \% & Média \% & Média \\
\hline GI & 0,0 & 0,0 & 0,0 & 0,0 & 2,5 & 7,5 \\
GII & 48,5 & 50,0 & 51,5 & 46,5 & 50,0 & 75,0 \\
GIII & 93,5 & 91,5 & 60,0 & 90,0 & 85,0 & 56,5 \\
GIV & 80,0 & 66,5 & 48,5 & 85,0 & 70,0 & 76,5 \\
GV & 65,0 & 58,5 & 36,5 & 50,0 & 56,5 & 88,5 \\
\hline
\end{tabular}

GI: Controle, GIl: Cipermetrina 15\% + Clorpiriphos 25\% + Citronela 1\%, GIII: Amitraz 12,5\%, GIV: Cipermetrina 15\%, GV: Deltametrina $25 \%$.

Larvas tratadas com amitraz 12,5\% (GIII) apresentaram significativa perda de motilidade na fase mais aguda, alcançando eficácia superior a 90,0\% nos períodos de 5 e 60 minutos após o tratamento (Tabela 2). Tal efeito observado foi descrito na literatura como "Knockdown", isto é, capacidade de causar paralisia motora rapidamente, derrubando os ixodídeos adultos presentes no animal logo após o tratamento (Soares et al., 2009). Porém observa-se que 24 horas após o tratamento, apenas $56,5 \%$ das larvas tratadas com amitraz $12,5 \%$ apresentavam perda de motilidade, confirmando o efeito tóxico transitório deste composto, fato este que permitiu a recuperação de quase 50 por das larvas intoxicadas. Tal efeito também foi também registrado por CASTELLÀ-SPUNY \& SOUZA (1998) para os princípios 
ativos amitraz, fipronil e permetrina avaliados in vitro contra larvas de Rhipicephalus bursa em Barcelona na Espanha.

Por outro lado, larvas expostas ao tratamento com deltametrina a $25 \%$ apresentaram eficácia tardia significativa resultando em $88,5 \%$ de larvas com perda de motilidade (Tabela 2), demonstrando ser entre os compostos o mais eficaz para controlar a presença de larvas no ambiente. Os tratamentos contendo outro piretróide sintético a cipermetrina (grupos II e IV) apresentaram eficácia de 75 e $76,5 \% 24$ horas após o tratamento. SILVA et al. (2010) verificaram efeito semelhante no controle de larvas de Rhipicephlus (Boophilus) microplus, ixodídeo de bovinos, pois entre ambos os piretróides a deltametrina também foi mais eficaz do que a cipermetrina. Tais achados confirmam o valor acaricida da deltametrina para 0 controle de larvas de ixodídeos no ambiente.

\section{Experimento II - Teste pacote de larvas (TPL)}

Pela técnica preconizada pela Organização das Nações Unidas (TPL), constatou-se, após o desafio, a eficácia na perda de motilidade de 90,0\% em larvas submetidas aos tratamentos com Cipermetrina 15,0\% associado ao organofosforado clorpirivos $25,0 \%$ e citronela1\% (GII) e 73,3\% quando tratadas com o outro piretróide deltametrina $25,0 \%$ (Tabela 3 ). Tais achados confirmam os resultados observados no estudo da pipeta (Experimento I), demonstrando que os piretróides representam uma boa estratégia para controle de larvas no animal e ambiente, sendo novamente a deltametrina o mais eficaz, pois apesar da cipermetrina $15 \%$ quando associada ao organofosforado mostrar mais eficácia a sua administração sozinha (Grupo IV) resultou em eficácia inferior a apresenta pela deltametrina, corroborando os achados de SILVA et al. (2010).

Em conformidade com o experimento I, o tratamento com amitraz $12,5 \%$ resultou em eficácia de $43,3 \%$ no controle da motilidade das larvas do Amblyomma cajennense (Tabela 3 e Figura 1), confirmando a baixa atividade deste ativo sobre 0 controle de larvas no ambiente. Tais achados corroboram as conclusões descritas por CASTELLÀ-SPUNY \& SOUZA (1998) ao estudarem o efeito deste acaricida sobre a perda de motilidade de larvas do Rhipicephalus bursa.

TABELA 3. Média percentual de redução de motilidade larvar e desvio padrão (DVP) de larvas de Amblyomma cajennense submetidas a diferentes formulações carrapaticidas.

\begin{tabular}{ccccc}
\hline \multirow{2}{*}{ Tratamento } & \multicolumn{4}{c}{ Avaliação de perda de motilidade com 24 horas } \\
\cline { 2 - 5 } & Média \% & DVP & Mínimo & Máximo \\
\hline GI & 0,0 & 0,00 & 100 & 100 \\
GII & 90,0 & 0,00 & 10 & 10 \\
GIII & 43,3 & 11,55 & 50 & 70 \\
GIV & 56,6 & 5,77 & 40 & 50 \\
GV & 73,3 & 5,77 & 20 & 30 \\
\hline
\end{tabular}

GI: Controle, GII: Cipermetrina 15\% + Clorpiriphos 25\% + Citronela 1\%, GIII: Amitraz 12,5\%, GIV: Cipermetrina 15\%, GV: Deltametrina $25 \%$. 


\section{CONCLUSÕES}

O estudo in vitro da eficácia larvicida de diferentes carrapaticidas sobre o ixodídeo de equinos, Amblyomma cajennense, demonstrou a maior eficácia do tratamento com deltametrina $25 \%$ no bloqueio da motilidade de larvas deste ácaro, confirmando a importância deste composto para o auxílio no controle ambiental de instares infestantes primários (larvas). O tratamento com amitraz $12,5 \%$ apresentou efeito tóxico imediato transitório sobre a motilidade das larvas, porém ocorrendo recuperação da capacidade motora, não sendo neste caso, recomendado para o uso no controle ambiental deste parasito.

\section{REFERÊNCIAS}

BARCI, L.A.G. \& NOGUEIRA, A.H.C. Método para avaliação de mortalidade de larvas de Boophilus microplus (Canestrini, 1887) submetidas a tratamentos com produtos carrapaticidas. Arquivos do Instituto Biológico, São Paulo, v.73, n.1, p.105-109, jan./mar., 2006.

BASSO, L. M. S.; MONTEIRO, A. C.; BELO, M. A. A.; SOARES, V. E.; GARCIA, M. V.; MOCHI, D. A.. Controle de larvas de Boophilus microplus por Metharizium anisopliae em pastagens infestadas artificialmente. Pesquisa Agropecuária Brasileira (1977. Impressa), v. 40, p. 595-600, 2005.

BELO, M. A. A.; PRADO, E.J.R.; SOARES, V. E.; SOUZA, L. M.; MOTA, F. C. C.; Giamlorenço, T.F.; GÍRIO, T.M.S.. Eficácia de diferentes formulações no controle da mosca Haematobia irritans em bovinos naturalmente infestados. Bioscience Journal (Online), v. 28, p. 245-250, 2012.

BRITES-NETO, J.; NIERI-BASTOS, F. A.; BRASIL, J.; DUARTE K.M.R.; MARTINS, T.F.; VERISSIMO, C.J.; BARBIERI, A.R.M.; LABRUNA, M.B.. Environmental infestation and rickettsial infection in ticks in an area endemic for Brazilian spotted fever. Revista Brasileira de Parasitologia Veterinária, Jaboticabal , v. 22, n. 3, p. 367-372, Sept. 2013.

BRITES-NETO, J.; DUARTE, K.M.R., MARTINS, T.F. Tickborne infections in human and animal population worldwide, Veterinary World 8(3): p.301-315, 2015.

CASTELLÀ ESPUNY, J \& SOUZA, L.M. Actividade in vitro del amitraz, friponil y permetrina, frente a larvas de Rhipicephalus bursa. In: Anais - VI Simpósio Ibérico sobre ixoididea e enfermedades transmisibiles, Setubal, Portugal, 24 a 26 de setembro, 1998.

CHACÓN, S.C.; CORREIA, P.G.; BARBIERI, F.S.; DAEMON, E.; JOÃO L.H. FACCINI, J.L.H. Efeito de três temperaturas constantes sobre a fase não parasitária de Amblyomma cajennense (FABRICIUS, 1787) (ACARI: IXODIDAE). Revista Brasileira de Parasitologia Veteterinária, v.12, 1, p.13-20, 2003.

CUNHA, A.P.; BELLO, A.C.P.; LEITE, R.C.; BASTIANETTO, E.; RIBEIRO, A.C.C.L.; FREITAS, C.M.; OLIVEIRA, P.R. Controle estratégico de Amblyomma cajennense (FABRICIUS, 1787) (ACARI: IXODIDAE) em eqüinos, Minas Gerais, Brasil. Revista Brasileira de Parasitologia Veterinária, 16, 4, 221-228, 2007. 
FARIA, V. P.; SOUZA, L. M. ; SILVA, I. C.; SOARES, V. E. ; BELO, M. A. A.; TORRENTE, A. C. G.; SILVA, J. ; MAZZONETTO, F.; CHAGAS, A. C. S.. Avaliação in vitro do efeito larvicida do extrato aquoso de Erva de Santa Maria (Chenopodium ambrosioides), sobre Rhipicephalus (Boophilus) microplus. In: V Congresso da Sociedade Paulista de Parasitologia, Guarulhos / SP. 2010.

KRAWCZAK, F.S.; NIERI-BASTOS, F.A.; NUNES, F.P.; JOÃO F SOARES, J.F.; MORAES-FILHO, J.; LABRUNA, M.B.: Rickettsial infection in Amblyomma cajennense ticks and capybaras (Hydrochoerus hydrochaeris) in a Brazilian spotted fever-endemic area. Parasites \& Vectors 7:7. 2014.

LABRUNA M.B, WHITWORTH T, HORTA M.C, BOUYER DH, McBRIDE J.W, PINTER A, et al. Rickettsia species infecting Amblyomma cooperi ticks from an area in the State of São Paulo, Brazil, where Brazilian Spotted Fever is endemic. J Clin Microbiol; 42(1): 90-98. 2004.

PIRES, M.S.; SANTOS, T.M.; SANTOS, H.A.; VILELA, E.C.R.; PEIXOTO, M.P.; ROIER, E.C.R.; SILVA, C.B.; BARREIRA, J.D.; LEMOS, E.R.S; MASSARD, C.L. Infestação por Amblyomma cajennense em equinos de duas microrregiões do estado do Rio de Janeiro, Brasil. Revista Brasileira de Parasitologia Veterinária., Jaboticabal, v. 22, n. 2, p. 235-242, abr.-jun. 2013.

SILVA, I. C.; SOUZA, L. M.; BELO, M. A. A.; SOARES, V. E.. Larval hatchability from eggs masses of Rhipicephalus (Boophilus) microplus treated with different acaricides. In: XIII International Congress of Acarology, Recife / PE, 2010.

SILVA, J. ; SOUZA, L. M.; SILVA, I. C.; SOARES, V. E.; BELO, M. A. A.; TORRENTE, A. C. G.; FARIA, V. P.; MAZZONETTO, F.; CHAGAS, A. C. S.. Potencial larvicida do extrato aquoso de Jatropha curcas (Pinhão manso), sobre Rhipicephalus (Boophilus) microplus. In: V Congresso da Sociedade Paulista de Parasitologia, Guarulhos / SP. 2010.

SOARES, V. E.; BELO, M. A. A.; SOUZA, L. M.; GUIARO, C. R.; BORTOLETTO NETO, O.; GIRIO, T. M. S.. Associação de cipermetrina, diclorvós e butóxido de piperonila contra Rhipicephalus (Boophilus) microplus em bovinos naturalmente infestados. Archives of Veterinary Science, v. 14, p. 1-8, 2009.

SOARES, V. E.; BELO, M. A. A.; REZENDE, P. B.; SOCCOL, V. T.; FUKUDA, R. T.; OLIVEIRA, G. P.; COSTA, A. J.. Distribuition of Taenia saginata metacestodes: comparison of routine meat inspection and carcase dissection results in experimentally infected calves. Annals of Tropical Medicine and Parasitology INCLUDEPICTURE "http://buscatextual.cnpq.br/buscatextual/images/curriculo/jcr.gif" I* MERGEFORMATINET, v. 105, p. 393-401, 2011.

SOUZA, L. M.; SILVA, G. S.; BELO, M. A. A.; SOARES, V. E.; COSTA, A. J.. Controle de Alphitobius diaperinus (Panzer) (Coleoptera: Tenebrionidae) em instalações avícolas de corte tratadas com spinosad. Archives of Veterinary Science, v. 14, p. 214-220, 2009. 
SINDHU, Z.-U.-D.; JONSSON, N.N.; IQBAL, Z. Syringe test (modified larval immersion test): A new bioassay for testing acaricidal activity of plant extracts against Rhipicephalus microplus. Veterinary Parasitology, v. 188, p. 362-367, 2012.

TORRENTE, A. C. G.; SOUZA, L. M.; SILVA, I. C.; SOARES, V. E.; BELO, M. A. A.; SILVA, J.; FARIA, V. P. ; MAZZONETTO, F.; CHAGAS, A. C. S.. Ação larvicida do extrato aquoso de Ocimum basilicum L (Alfavaca) sobre Rhipicephalus (Boophilus) microplus. In: V Congresso da Sociedade Paulista de Parasitologia, Guarulhos/SP, 2010. 